\title{
A Computational Framework for Proteome-Wide Pursuit and Prediction of Metalloproteins using ICP-MS and MS/MS Data
}

W Andrew Lancaster ${ }^{1 \dagger}$, Jeremy L Praissman ${ }^{1 \dagger}$, Farris L Poole $\|^{1}$, Aleksandar Cvetkovic ${ }^{1}$, Angeli Lal Menon ${ }^{1}$, Joseph W Scott ${ }^{1}$, Francis E Jenney $\mathrm{Jr}^{1,2}$, Michael P Thorgersen ${ }^{1}$, Ewa Kalisiak ${ }^{3}$, Junefredo V Apon ${ }^{3}$, Sunia A Trauger ${ }^{3}$, Gary Siuzdak ${ }^{3}$, John A Tainer ${ }^{4}$, Michael W W Adams ${ }^{1 *}$

\begin{abstract}
Background: Metal-containing proteins comprise a diverse and sizable category within the proteomes of organisms, ranging from proteins that use metals to catalyze reactions to proteins in which metals play key structural roles. Unfortunately, reliably predicting that a protein will contain a specific metal from its amino acid sequence is not currently possible. We recently developed a generally-applicable experimental technique for finding metalloproteins on a genome-wide scale. Applying this metal-directed protein purification approach (ICPMS and MS/MS based) to the prototypical microbe Pyrococcus furiosus conclusively demonstrated the extent and diversity of the uncharacterized portion of microbial metalloproteomes since a majority of the observed metal peaks could not be assigned to known or predicted metalloproteins. However, even using this technique, it is not technically feasible to purify to homogeneity all metalloproteins in an organism. In order to address these limitations and complement the metal-directed protein purification, we developed a computational infrastructure and statistical methodology to aid in the pursuit and identification of novel metalloproteins.
\end{abstract}

Results: We demonstrate that our methodology enables predictions of metal-protein interactions using an experimental data set derived from a chromatography fractionation experiment in which 870 proteins and 10 metals were measured over 2,589 fractions. For each of the 10 metals, cobalt, iron, manganese, molybdenum, nickel, lead, tungsten, uranium, vanadium, and zinc, clusters of proteins frequently occurring in metal peaks (of a specific metal) within the fractionation space were defined. This resulted in predictions that there are from 5 undiscovered vanadium- to 13 undiscovered cobalt-containing proteins in Pyrococcus furiosus. Molybdenum and nickel were chosen for additional assessment producing lists of genes predicted to encode metalloproteins or metalloprotein subunits, 22 for nickel including seven from known nickel-proteins, and 20 for molybdenum including two from known molybdo-proteins. The uncharacterized proteins are prime candidates for metal-based purification or recombinant approaches to validate these predictions.

Conclusions: We conclude that the largely uncharacterized extent of native metalloproteomes can be revealed through analysis of the co-occurrence of metals and proteins across a fractionation space. This can significantly impact our understanding of metallobiochemistry, disease mechanisms, and metal toxicity, with implications for bioremediation, medicine and other fields.

\footnotetext{
* Correspondence: adams@bmb.uga.edu

† Contributed equally

'Department of Biochemistry and Molecular Biology, University of Georgia,

Athens, GA 30602, USA

Full list of author information is available at the end of the article
} 


\section{Background}

Metallomics is an emerging field that seeks to comprehensively characterize the role of metals in organisms [1]. As with any new field, unique challenges have been encountered in terms of experimental methodologies and data analysis. The essential role metals play in biology has long been appreciated, but the complete metallome of any organism has yet to be characterized. It is estimated that around a third of all proteins in an organism require a metal partner [2]. While it is possible to predict that certain proteins contain a metal [3], there are fundamental limitations to all current computational methods in comprehensively describing the metalloproteome of any organism. Many current methods rely on sequence motifs that in turn depend on the existence of a sufficiently sized set of previously annotated homologous proteins, a problem further compounded by the diversity of metal-binding sites across organisms as well as within a single organism [4]. It is also well known that heterologous protein expression can result in the production of proteins incorporating metals that are not natively incorporated [5]. In addition, while certain proteins and protein families are known to bind a variety of metals and are annotated accordingly, many are annotated as binding a single metal based on limited evidence.

It was shown recently [6] that the set of metals known to interact with proteins in vivo is a significant underestimate of the true extent and diversity of the metalloproteome. The approach developed used metal-directed protein purification relying on inductively coupled plasma mass spectrometry (ICP-MS) and tandem mass spectrometry (MS/MS) and revealed that a prototypical microbe, Pyrococcus furiosus, takes up 21 of 53 metals measured in its growth medium, 18 of which are present in macromolecular complexes. These results are in stark contrast to the five metals that had previously been identified in proteins individually purified from the same organism. Further, of the 343 metal peaks found across the fractions from a second level of chromatography fractionation (for the 10 metals that were detected), almost half (158) contained no known or predicted metalloprotein corresponding to that particular metal [6]. The purification of eight of these metal peaks resulted in the identification of novel metalloproteins, or proteins containing unexpected metal ions [6]. Unfortunately, this method has two major limitations. Firstly, given the large number (158) of unassigned metal peaks and difficulty in purifying a single protein, it is impractical to purify a significant portion of these novel metalloproteins. Secondly, it is not technically feasible to natively purify proteins of very low abundance over several chromatographic steps.

Herein is described a computational infrastructure and analytical methodology developed to both aid in the pursuit of novel metalloproteins [7] as well as to predict which proteins observed via MS/MS during this fractionation are likely to be metalloproteins without requiring purification to homogeneity. This required the development of a database, an Online Analytical Processing (OLAP) cube and InterPro-Metal (IPM) automated metal domain identification methods (directly supporting the pursuit of novel metalloproteins), as well as a Global Metal Protein Association (GMPA) analysis (enabling the prediction of metal-protein associations without complete purification). Given the essential biological role of metals, the discovery of novel metalloproteins has a multitude of implications in a variety of fields $[1,8]$. Moreover, the computational infrastructure and methods described can be applied to any form of biomass of interest from tissues to microbes to identify potential metalloprotein targets for experimental characterization. Most importantly, this analysis allows the discovery of low abundance metalloproteins without radioisotope labeling, which have eluded other methods $[9,10]$ but which nonetheless may occupy key roles in essential biological pathways.

\section{Methods}

\section{Experimental design}

The experimental data set utilized in this study is an expanded version of the data set described in [7]. Briefly, native biomass of the hyperthermophilic archaeon Pyrococcus furiosus was fractionated anaerobically through multiple non-denaturing chromatography steps utilizing multiple column chemistries. The resulting 2,589 fractions were analyzed by ICP-MS to identify metals, and by MS/MS to identify proteins (primarily high-throughput MS/MS in this study). The MS/MS data were filtered such that the false discovery rate was less than $1 \%$, as described in [7] and only proteins identified by Mascot with two or more peptides were considered in the current bioinformatics based study. The use of nondenaturing native chromatography, ICP-MS and MS/MS captures the co-occurrence of metals and proteins in their native form, and enabled a metal-based purification strategy, in contrast to conventional enzyme assay guided protein purification [6]. While this metal-based separation was used to purify a number of metalloproteins to homogeneity [6], the wealth of metal and protein data collected for proteins that were not explicitly targeted for direct purification provided an additional opportunity (applying data analysis techniques) to identify proteins that are likely to contain one or more metals in their native form.

\section{Data infrastructure}

A relational database was constructed using Microsoft SQL Server 2005 to store the data used in this study. 
The database consists of three principal modules: a procedural (fractionation) module, a metal data (ICP-MS) module, and a protein data (MS/MS) module. The fractionation module was designed to store the procedural information used in each of the separation steps carried out during the multi-level column fractionation (allowing reconstruction of the complete experimental pathway). These multi-level hierarchical relationships between fractions were queried using recursive common table expressions (CTE). The metal data module was designed to store both procedural data and replicate metal concentration data for each sample and metal analyzed using ICPMS. This module also stores the peak assignments determined by manual inspection. Finally, the protein module stores data for each peptide identified by MS/MS, its protein source and the corresponding ORF, and details related to the MS/MS run and Mascot search as imported from Mascot XML result files. All fractions and samples (from fractions) collected were assigned unique IDs and labeled with $2 \mathrm{D}$ data matrix barcodes to facilitate sample tracking. This ensured the simple and reliable association of the data contained in all three principal modules within the context of the experimental hierarchy.

The relational database contains 2,589 records corresponding to fractions (the fractionation module) obtained from the chromatographic separation in which 1,026 proteins (the protein module) were identified by MS/MS. Of these 1,026 proteins (corresponding to 135,989 peptide hit records in the database), 870 were identified with 2 or more peptides $(125,777$ peptide hit records) and were used in statistical scoring of metal association (GMPA scores, see below). Each of these fractions has associated metal concentration data generated by ICP-MS analysis for multiple metals depending in part on what metals were relevant to the goals of a given separation step (up to 53 with 78,514 overall metal concentration records; the metal module). An Online Analytical Processing (OLAP) cube (Figure 1, middle right) was constructed on top of the relational database using Microsoft SQL Server 2005's Analysis Services. The cube enabled efficient preprocessing of significant amounts of aggregate data (e.g. sums, averages, etc.) and at the same time enabled convenient data slicing across multiple dimensions of experimental data in real-time (e.g. queries as to the number of proteins and metals detected in the same fraction) [11]. OLAP is commonly used in the business analysis field but has not been widely exploited in scientific fields. However, OLAP is particularly well suited to the types of analyses presented herein given the hierarchical nature of the data set and the aggregate nature of queries utilized in this study to investigate experimental outcomes (e.g. are we purifying a protein-i.e. are metal/protein ratios improving?). A more detailed description of data infrastructure is given in the supplementary material (Additional Files 1, 2, 3, 4, 5). For the 870 proteins with GMPA scores, metal concentrations and numbers of peptides in the column fractions containing those proteins (designated by ORF number) are available at http://enigma.bmb. uga.edu/impact.

\section{Bioinformatic metalloprotein prediction-InterPro-Metal (IPM) automated metal domain analysis}

The set of known metalloproteins that have been previously purified and characterized from $P$. furiosus by conventional chromatographic methods consists of only 23 proteins (encoded by 39 genes). Each contains one or more of $\mathrm{Co}, \mathrm{Fe}, \mathrm{Ni}, \mathrm{W}$ and/or $\mathrm{Zn}$ atoms [6]. Although the utility of bioinformatic predictions is limited, such predictions can be used to identify homologs of more extensively characterized metalloproteins and serve as a starting point for assigning proteins to the observed metal peaks. The Integrated Resource of Protein Domains and Functional Sites (InterPro) [12] was used to predict known metal associated domains encoded in the genome of $P$. furiosus. InterPro integrates multiple popular protein feature databases, and provides the Iprscan utility for searching protein sequence queries against these databases. The genome was searched using this utility and the resulting matches of proteins to InterPro entries were stored in a relational database (protein-InterPro data). The description of each InterPro entry, including name, abstract and publication list, is available in a downloadable XML file ftp://ftp.ebi.ac.uk/pub/databases/ interpro/interpro.xml.gz. This file was parsed and inserted into a corresponding relational database. A number of regular expression patterns relating to metal ions, metal cofactors and metal binding domains were used to search the text of each InterPro entry description to classify the entries which potentially involve specific metals (metal-InterPro data). Those metal-InterPro entries that had hits in the P. furiosus genome were evaluated manually for quality and assigned a subjective score. In some cases, keyword hits were not deemed to be indicative of a potential association of the given domain with a given metal, for instance an abstract for a particular subfamily of an enzyme may include additional information on other subfamilies which use alternate metals. Such spurious hits were assigned a score of 0 while metals with evidence of association with the given domain were assigned a positive score. All hits with nonzero scores were considered as potentially metal associated domains in subsequent analyses. The protein-InterPro data and metal-InterPro data were joined to determine 


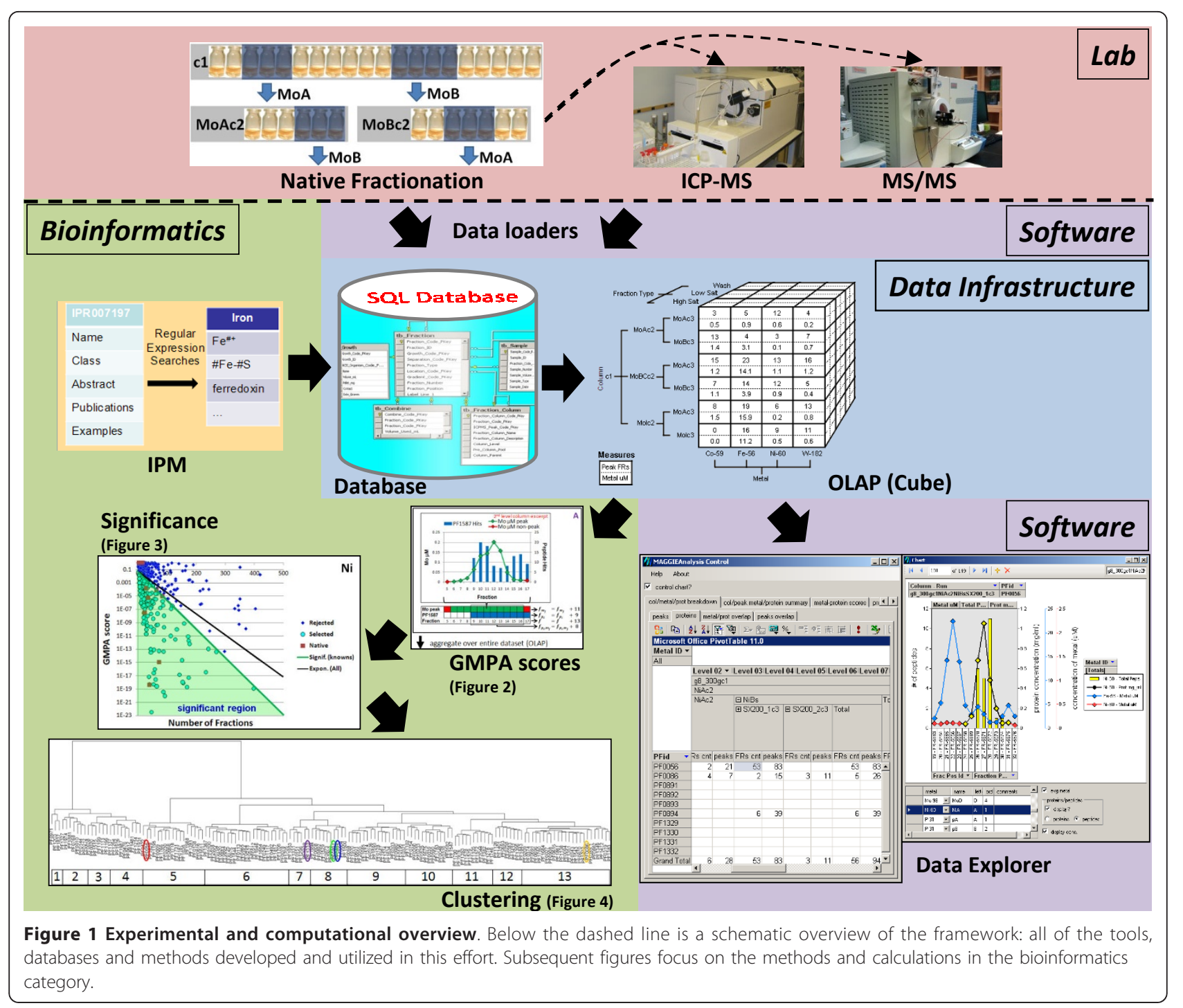

which $P$. furiosus proteins had associations with specific metals and this subsequently will be referred to as the InterPro-Metal (IPM) database or InterPro-Metal analysis. These domain-based predictions were incorporated into the relational database and OLAP cube (Figure 1) to aid in the identification of novel metalloproteins and proteins for which the metal prediction and observed metal associations differ.

\section{Data driven metalloprotein prediction-Global Metal Protein Association (GMPA) analysis}

The previously described infrastructure enables efficient querying of the data along the following dimensions: protein identity, metal motif prediction for identified proteins, total protein concentration and metal concentration across the entire experimental data set. This database enables an evaluation of the global association of metals with proteins across the entire observed space. The heterogeneity of the utilized data, with ICP-MS yielding quantitative metal concentrations and the MS/MS results indicating the presence or absence of proteins (with peptide counts providing only local semi-quantitative comparison), dictated the use of methods which are less reliant on quantitative agreement between these data sets. Metal peak fraction regions were defined and entered into the database manually based roughly on the presence of at least two fractions in which the concentrations for a given metal (as indicated by two independent ICP-MS technical replicates) were substantially above the surrounding data for that metal. A hypergeometric distributionbased statistic that only considers the presence of proteins in these metal peaks instead of similarity of the shape or size of the metal and protein peaks (e.g. peptide counts of a given protein across a relatively contiguous set of fractions) was utilized. This statistic, the 
"Global Metal Protein Association score" (GMPA score, $\left.\mathrm{G}\left(\mathrm{p}_{\mathrm{i}}, \mathrm{m}_{\mathrm{j}}\right)\right)$ is defined as:

$$
\begin{aligned}
& G\left(p_{i}, m_{j}\right)=\sum_{n=\max \left(f_{p_{i}, m_{j}}, f_{p_{i}}-\left(f-f_{m_{j}}\right)\right)}^{\min \left(f_{p_{i}}, f_{m_{j}}\right)} \quad\left(\begin{array}{c}
f_{m_{j}} \\
n
\end{array}\right)\left(\begin{array}{c}
f-f_{m_{j}} \\
f_{p_{i}}-n
\end{array}\right) \\
& =\operatorname{phyper}\left(f_{p_{p_{i}}, m_{j}}-1, f_{m_{j^{\prime}}}, f-f_{m_{j^{\prime}}}, f_{p_{p^{\prime}}}, \text { FALSE }\right)
\end{aligned}
$$

where $f=$ the number of fractions in the data set, $f_{m_{j}}=$ the number of fractions inside of peaks of metal $j$ in the data set, $f_{p_{i}}=$ the number of fractions in which protein $i$ was observed, and $f_{p_{i}, m_{j}}=$ the number of fractions in which protein $i$ was observed that are also contained within peaks of metal $j$. This statistic gives the probability of at least $f_{p_{i}, m_{j}}$ fractions out of the $f_{p_{i}}$ fractions in which protein $i$ is found occurring within peaks of metal $j$ assuming that the $f_{p_{i}}$ fractions were distributed randomly (with uniform probability) across the fraction space (Figure 2). The GMPA score was computed for all data sets we examined using the phyper function in R-2.10.1 [13] according to the formula above. The lower the score for a given protein/metal combination, the less likely it is that the protein and metal co-occur in the chromatographic fraction space by chance.

The frequency of observation plays a large role in the GMPA score, so an exponential significance curve through the GMPA score/occurrences space was introduced dividing it into a set of proteins with substantial evidence for metal association (lower GMPA scores, fewer fraction occurrences) and a set without as much evidence (higher GMPA scores, more fraction occurrences). This provides an initial filtering step for our current real-world data set (Figure 3). For metals significantly represented in the set of known metalloproteins $(\mathrm{Fe}, \mathrm{Ni}$, and $\mathrm{W})$, the significance curve was generally set using these known references. For metals present in few or no known metalloprotein(s), the curve was extrapolated from the sets with sufficient known metalloproteins (Additional File 6). This extrapolation was based on the ratios between the exponent of an exponential regression curve calculated over all proteins through the GMPA score/occurrences space (Figure 3) and the significance curves chosen to capture all known metalloproteins that could reasonably be captured for $\mathrm{Fe}, \mathrm{Ni}$ and W. Typically, an absolute GMPA score cut-off was employed after significance curve filtering to remove proteins that occurred in fewer than approximately 10 fractions from further analysis since it is unlikely that anything can be determined from our data with any confidence for such proteins.

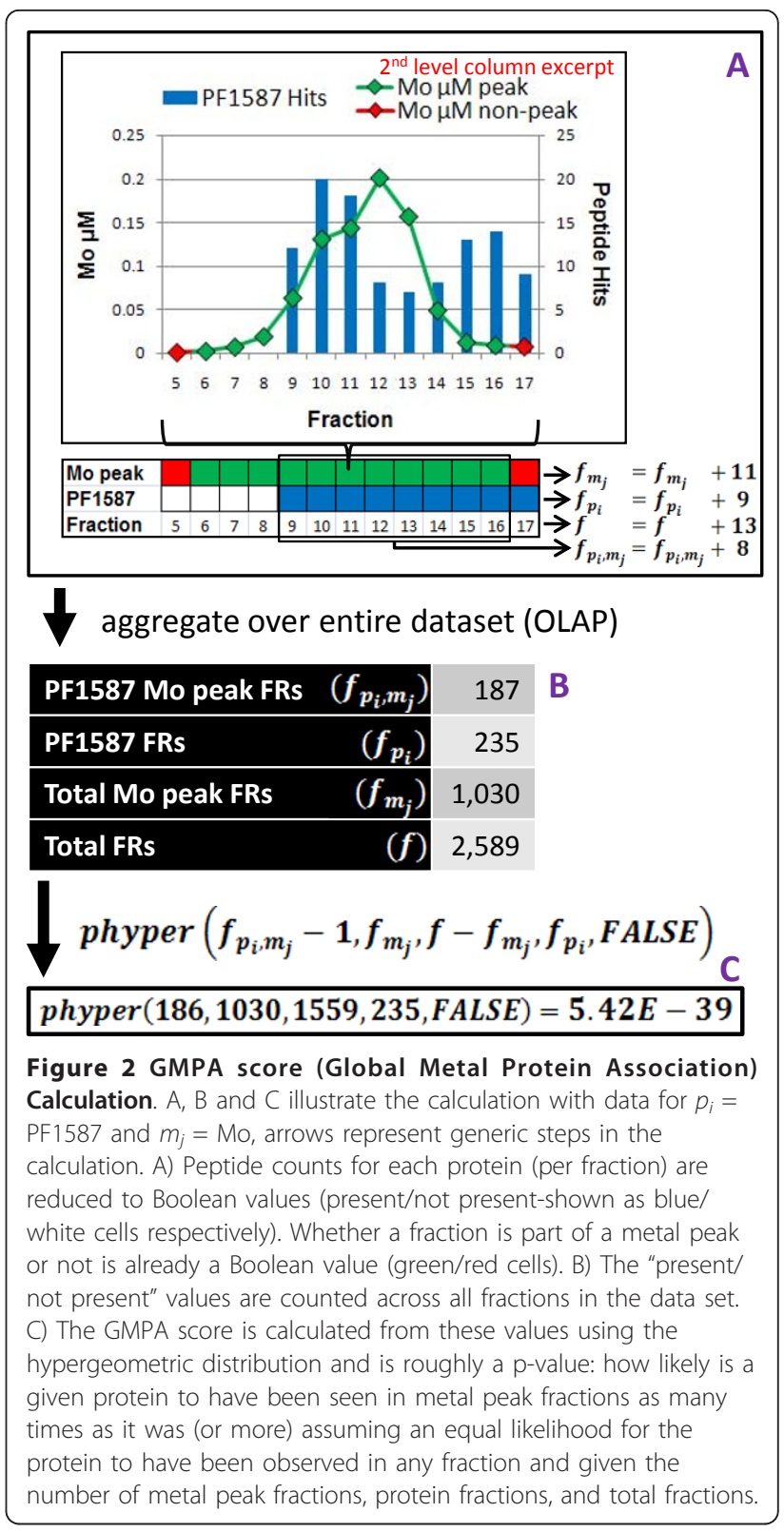

To further organize the space of potential metalloproteins and to estimate the number of metalloproteins that could reasonably be expected from among the set of GMPA score filtered proteins for each metal, hierarchical clustering was applied. Hierarchical clustering organizes the elements of a set into a tree based on similarity (co-occurrence of proteins in fractions in this case). The resulting tree can facilitate the partitioning of the original set into sub-tree "clusters" capturing natural divisions in the data. This technique is particularly useful when the number of natural groups that might exist in the data is not known a priori. Ward's method of hierarchical clustering, which is variance-minimizing, along with the Euclidean metric and the Dynamic Tree 


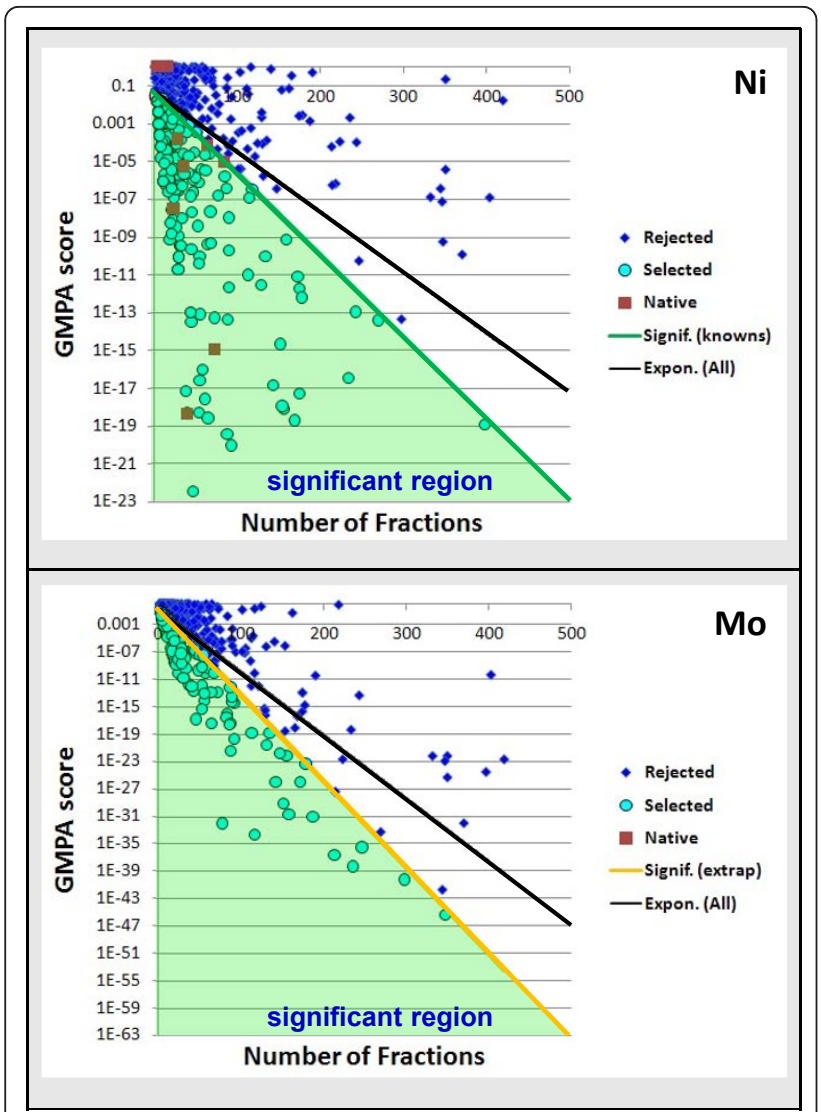

Figure 3 Protein selection using GMPA score significance curve criterion. Scatterplots (per metal) of the GMPA scores versus number of fraction occurrences for all proteins. The green/orange lines are exponential "significance" curves (plotted on a logarithmic scale) and proteins below are considered significant and selected for clustering (green regions and cyan points). The Ni significance curve (green) was based on occurrences of known Ni-proteins (red points) and the Mo significance curve (orange) was extrapolated from the relationships between the significance curves and exponential regression curves (through all points-the black lines) on average across all metals with known metalloproteins. Typically, this step removes an additional 20-35 proteins beyond what would be removed using the regression curves themselves.

Cut package for $\mathrm{R}$ [14] were found to give useful selfcontained clusters. The cutreeHybrid function of the Dynamic Tree Cut package was used to analyze the trees generated for each metal (Figure 4, Additional Files 7 and 8 -graphics produced in part using slightly modified versions of functions from [15]). The parameters passed to this function were tuned for Mo and $\mathrm{Ni}$ so that within the selected clusters there were clear "core" regions (composed of fractions with $\geq 50 \%$ of proteins in the cluster observed) while "core" region overlap was minimal between pairs of clusters. Low overlap between clusters in metal peak regions, with which core regions typically coincide, makes it likely that each cluster should contain at least one metalloprotein in order to explain the metal peak data-i.e. that the dimensionality reduction achieved in the clusterings is reasonable. Approximate minima required to cover all peaks were also calculated using the greedy algorithm approach to the set cover problem [16] and were found to be consistent with the results of clustering. The parameters found for Mo and Ni were then applied for all metals.

\section{Results and Discussion}

The computational framework that was developed consisted of a database, an Online Analytical Processing (OLAP) cube, InterPro-Metal (IPM) automated metal domain identification and Global Metal Protein Association (GMPA) analysis. This complemented and enhanced our recent effort to elucidate the metalloproteome of $P$. furiosus and to identify novel metalloproteins [6]. The GMPA analysis in particular was used to provide estimates of the numbers of metalloproteins that could be expected proteome-wide and a narrowed list of candidates (based on ICP-MS and MS/MS data) at various stages of the column chromatography fractionation, culminating in our predictions at the conclusion of the study that the numbers of undiscovered metal containing proteins in $P$. furiosus range from approximately 5 for vanadium up to as many as 13 for cobalt. Validation of these predictions is provided by the overall success of the GMPA analysis in categorizing known metalloproteins from P. furiosus, the establishment of lower bounds on the numbers of proteins required to explain all metal peaks seen during the fractionation, the fact that the predictions lie within reasonable ranges in the context of literature (where up to a third of proteins are expected to contain or be involved with metals in various ways with the caveat that the majority are likely to be involved with $\mathrm{Mg}$ ) $[2,17,18]$ and considering the effect of dynamic association/adventitious metal binding [19].

In order to determine how much of the information contained in our data set remains uncaptured by the GMPA analysis, nickel (Ni) and molybdenum (Mo) were chosen for manual evaluation using the GMPA predictions as a starting point. Of the 870 proteins identified by MS/ MS with two or more peptides, 153 and 119 were found to be significantly associated with $\mathrm{Ni}$ and Mo respectively, upon clustering yielding predictions of 13 and 10 total Niand Mo-proteins in the proteome of $P$. furiosus. The local semi-quantitative MS/MS peptide hit data for each of the proteins clustered was then manually compared to the local metal concentration data using our data explorer (Figure 1). This step excluded an additional 131 and 99 proteins producing top candidates lists of 22 and 20 proteins that are most likely to contain $\mathrm{Ni}$ and Mo respectively. These lists were then analyzed more extensively through bioinformatic analyses and literature searches. 


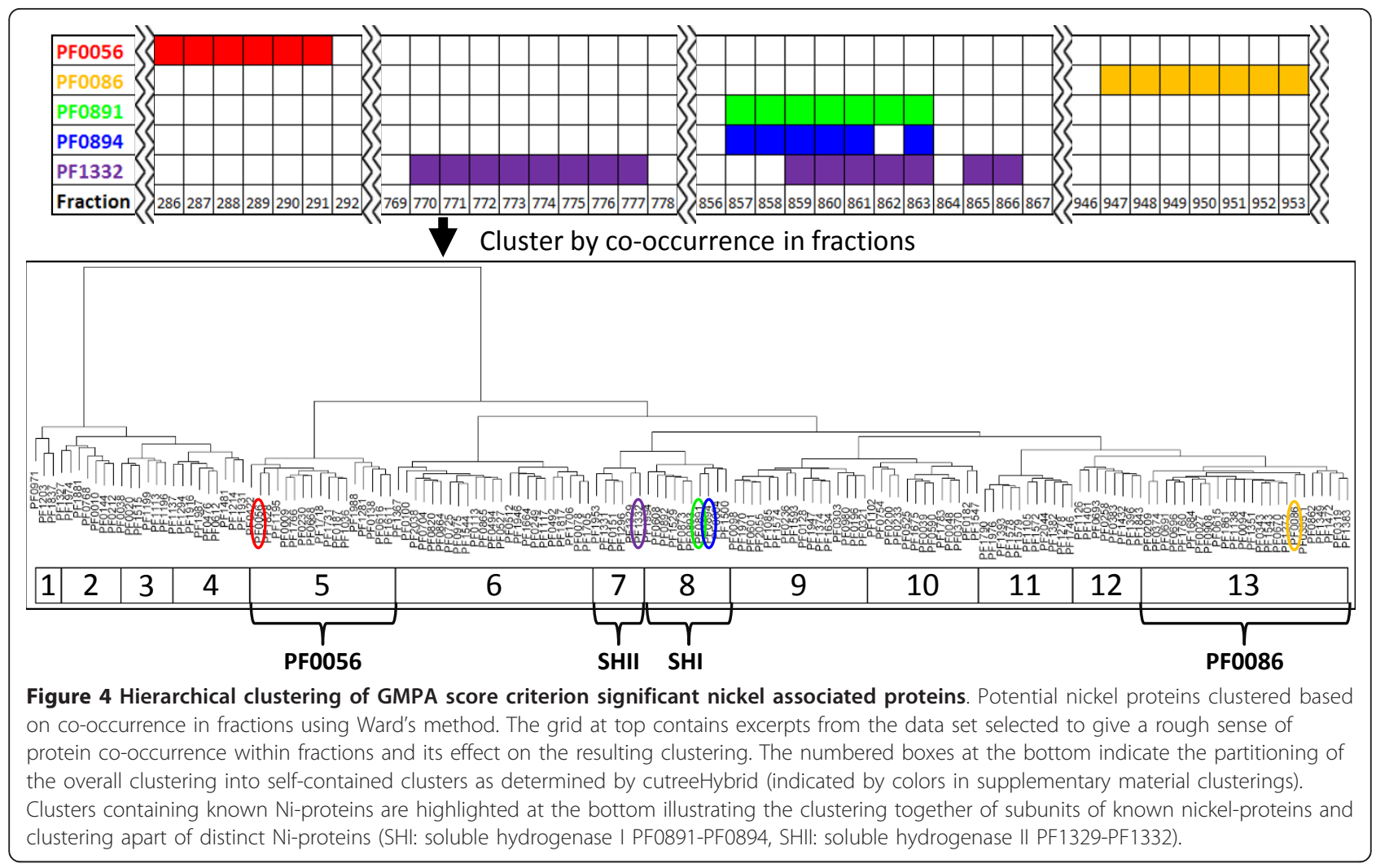

We will first describe the results obtained at the conclusion of our experimental study and then discuss the bioinformatics of the lists of predicted $\mathrm{Ni}$ - and Mo-proteins, concluding with the limitations inherent in this study.

\section{Bioinformatic metalloprotein prediction-InterPro-Metal (IPM) automated metal domain analysis predictions} Of the 2,065 annotated opening reading frames (ORFs) in the RefSeq annotation of the P. furiosus genome [20], 376 were found to have matches to metal-associated InterPro entries. These included all of the 23 previously known metalloproteins [6]. Of the 376, 221 were detected by MS/MS with 2 or more peptides, 43 of which had matches to multiple metals, either from matches to multiple InterPro entries, or to a single InterPro entry that lists potential associations with multiple metals. Consistent with expectation, the majority of the $P$. furiosus proteins with metal-related InterPro hits were predicted to be associated with Fe or Zn, with fewer Mn, Mo, Co, W and $\mathrm{Ni}$ predictions (Figure 5) $[4,17]$. There were no predictions of association with lead or uranium other than transport proteins whose InterPro descriptions may list many metal ions. The observation of a metal peak in a fraction in which no predicted metalloprotein was identified shows that one of the proteins identified is a completely novel metalloprotein, or one which uses a metal other than that expected by its annotation [6].
An unexpectedly large number-158 of the 353 metal peaks detected for the second level of column fractionation-were found in the data set.

\section{Data driven metalloprotein prediction-Global Metal} Protein Association (GMPA) analysis predictions The 870 proteins identified by MS/MS with two or more peptides were assigned GMPA scores and partitioned

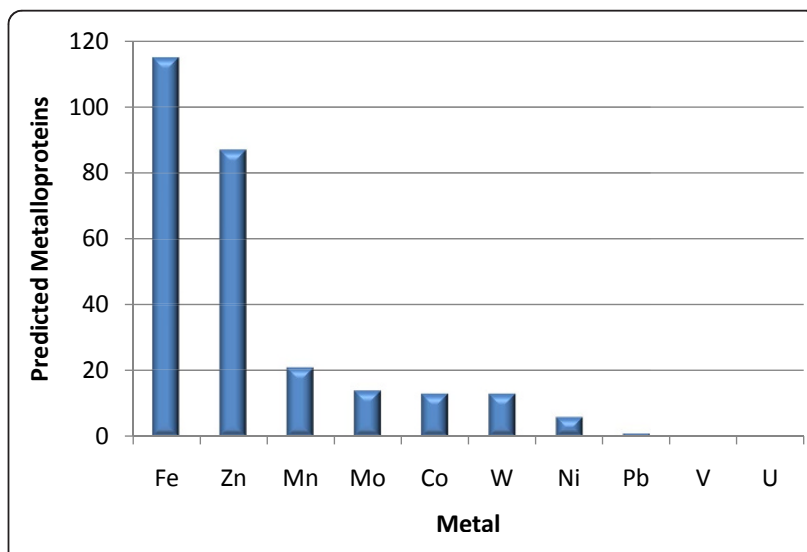

Figure 5 IPM predicted metalloproteins identified by MS/MS. Out of the 870 proteins identified with two or more peptides, 221 were predicted to be metalloproteins. The majority were predicted to contain Fe or $\mathrm{Zn}$ with fewer predicted to contain $\mathrm{Mn}, \mathrm{Mo}, \mathrm{Co}, \mathrm{W}$ and $\mathrm{Ni}$. 
into significant and insignificant regions as described in the methodology section. The number of proteins deemed significant ranged from 45 for $\mathrm{V}$ to 153 for $\mathrm{Ni}$ (Table 1). The proteins falling in the significant regions for each metal were then hierarchically clustered by co-occurrence in fractions, with the number of clusters ranging from 5 for $\mathrm{V}$ to 16 for Co (Table 1). Each of these clusters is assumed to contain at least one metalloprotein giving rise to the observed metal peaks (see Methods). A total of 23 metalloproteins are known for P. furiosus from previous studies [6], and the coverage of the corresponding clusterings by these standards, together with the metalloproteins discovered by metal-directed purification [6], is summarized in Table 1. As a specific example of the data underlying each row of this table, Figure 4 shows the 13 clusters into which the 153 significant (by GMPA score) Ni targets fell. The two known Ni-containing enzymes, the soluble hydrogenases, lie in distinct clusters, as illustrated in Figure 4.

\section{Nickel- and Molybdo-protein evaluation}

Nickel and molybdenum were selected for further detailed analysis primarily using a data explorer developed in-house leveraging the speed of the OLAP cube (Figure 1). There are 12 genes that encode subunits of known Ni-containing proteins in P. furiosus. A total of seven of the 12 proteins encoded by these genes were detected by MS/MS analyses including five genes that encode the two multi-subunit $\mathrm{Ni}$-containing soluble hydrogenases (I and II) of P. furiosus. These seven genes represent a set of positive controls for evaluation of the analysis. Prior to the metal-targeted comprehensive protein purification [6], P. furiosus was not known to have any Mo-proteins, so all proteins identified by that analysis represent novel Mo-proteins in this organism. For $\mathrm{Ni}$, out of 870 proteins observed by MS/MS in at least one fraction of the fraction-space used for this analysis, 153 proteins were selected in the initial GMPA score significance curve screening. The parameters for the significance curve for $\mathrm{Ni}$ were chosen directly based on the known $\mathrm{Ni}$-proteins such that the significance curve did not filter out any of the subunits of the Ni-containing soluble hydrogenase I (PF0891-PF0894) and only filtered out one of the subunits of the Ni-containing soluble hydrogenase II (PF1330). This is essentially unavoidable given that PF1330 was found in relatively few (16) fractions. From the 153 proteins, 13 reasonably distinct clusters were defined after hierarchical clustering and each was manually evaluated (Table 2 , Additional File 9). Seven of the clusters were found to contain proteins exhibiting local agreement of MS/MS data to the Ni ICP-MS data and 22 proteins or subunits of proteins were found in total (Table 2). Five of the 22 best candidates for $\mathrm{Ni}$ are in fact subunits of previously known Ni-protein complexes (Table 2 ) providing validation of this approach. An additional 2 of the 22 candidate Ni-proteins were very recently taken to purity or partial purity (PF0056 and PF0086) by the metaldirected purification and their assignment was confirmed [6]. This leaves 15 potential novel $\mathrm{Ni}$-containing proteins on which to carry out further experiments.

For Mo, out of 870 proteins observed by MS/MS in at least one fraction of the fraction-space used for this analysis, 119 proteins were selected in the initial GMPA score significance curve screening. The significance curve for Mo was selected (as described in Methods) by extrapolation based on the significance curves chosen for metals with known metalloproteins. From the 119 proteins,

Table 1 GMPA Analysis Clustering and Coverage of Known Metalloproteins

\begin{tabular}{|c|c|c|c|c|c|c|}
\hline \multirow{3}{*}{$\begin{array}{l}\text { Metal } \\
\text { Co }\end{array}$} & \multicolumn{3}{|c|}{ Known metalloprotein subunits } & \multirow{2}{*}{$\begin{array}{l}\text { Proteins } \\
\text { Clustered }\end{array}$} & \multicolumn{2}{|c|}{ Clusters } \\
\hline & Total & Observed & $\begin{array}{l}\text { Met GMPA } \\
\text { significance } \\
\text { criterion }\end{array}$ & & Total & $\begin{array}{l}\text { With known } \\
\text { metalloproteins }\end{array}$ \\
\hline & 5 & 5 & 3 & 139 & 16 & 3 \\
\hline $\mathrm{Fe}$ & $35(20)$ & 35 & 19 & 148 & 15 & 8 \\
\hline $\mathrm{Mn}$ & 0 & 0 & 0 & 73 & 7 & 0 \\
\hline Mo & 2 & 2 & 1 & 119 & 10 & 1 \\
\hline $\mathrm{Ni}$ & $12(5)$ & 12 & 9 & 153 & 13 & 4 \\
\hline $\mathrm{Pb}$ & 0 & 0 & 0 & 90 & 9 & 0 \\
\hline U & 0 & 0 & 0 & 76 & 7 & 0 \\
\hline V & 0 & 0 & 0 & 45 & 5 & 0 \\
\hline w & 5 & 5 & 4 & 136 & 11 & 4 \\
\hline $\mathrm{Zn}$ & 5 & 5 & 1 & 116 & 9 & 1 \\
\hline
\end{tabular}

Some proteins consist of multiple subunits, the number of holoenzymes are given in parentheses. Some proteins contain multiple metals and factor into multiple rows. For full clusters, refer to supplementary material. 
Table 2 Manually Evaluated Nickel Protein Candidates

\begin{tabular}{|c|c|c|c|c|c|}
\hline $\begin{array}{l}\text { Cluster } \\
\text { number }\end{array}$ & ORF & Annotation & $\begin{array}{c}\text { Crystal } \\
\text { structure } \\
\text { homolog }\end{array}$ & $\begin{array}{c}\text { Metal } \\
\text { In } \\
\text { structure }\end{array}$ & $\begin{array}{c}\text { IPM } \\
\text { prediction }\end{array}$ \\
\hline 2 & PF0144 & Aldolase-type TIM barrel & & & $\mathrm{Fe}$ \\
\hline 2 & PF1881 & Alba archaeal DNA/RNA-binding protein & $2 Z 7 C$ & & \\
\hline 3 & PF0038 & Beta-lactamase-like glyoxalase II family member & & & $\mathrm{Zn}$ \\
\hline 4 & PF1916 & Glycosyl transferase, family 2 & & & \\
\hline 4 & PF1987 & Conserved hypothetical protein & & & \\
\hline 5 & PF0056 & Carbohydrate binding protein & $1 \mathrm{VJ} 2$ & $M n$ & $M n$ \\
\hline 5 & PF0138 & Uncharacterized rubrerythrin domain protein & $2 \mathrm{FZF}$ & & $\mathrm{Fe}$ \\
\hline 6 & PF1664 & Phosphoribosyl-AMP cyclohydrolase & 1ZPS & $\mathrm{Cd}$ & Zn \\
\hline 6 & PF2038 & Adenosylcobalamin biosynthesis & $1 \mathrm{G} 5 \mathrm{~T}$ & $\mathrm{Mg}$ & Co \\
\hline 7 & PF1329 & Hydrogenase II beta & & & $\mathrm{Fe}$ \\
\hline 7 & PF1331 & Hydrogenase II delta & & & $\mathrm{Fe}$ \\
\hline 7 & PF1332 & Hydrogenase II alpha & & & $\mathrm{Fe}, \mathrm{Ni}$ \\
\hline 8 & PF0891 & Hydrogenase I beta & & & $\mathrm{Fe}$ \\
\hline 8 & PF0894 & Hydrogenase I alpha & & & $\mathrm{Fe}, \mathrm{Ni}$ \\
\hline 8 & PF1500 & PRC-barrel-like & & & \\
\hline 8 & PF1529 & Pyroxidine biosynthesis protein & $2 Y Z R$ & & \\
\hline 12 & PF1401 & Peptidyl-prolyl cis-trans isomerase & & & \\
\hline 13 & PF0086 & Alanyl-tRNA synthetase, class IIc & $2 \mathrm{E} 1 \mathrm{~B}$ & $Z n$ & \\
\hline 13 & PF0615 & Hydrogenase expression/formation protein A & $3 \mathrm{~A} 43$ & $\mathrm{Zn}$ & $\mathrm{Ni}$ \\
\hline 13 & PF1272 & LamB/YcsF & 1V6T & & \\
\hline 13 & PF1684 & Acetylglutamate kinase & $2 \mathrm{EGX}$ & & \\
\hline 13 & PF1861 & Lysyl aminopeptidase & 2PE3 & & $\mathrm{Zn}$ \\
\hline
\end{tabular}

Cluster number refers to hierarchical clustering with dynamic hybrid partitioning, see Figure 4 for explanation and supplementary tables 1-8 in Additional File 9 for complete cluster tables. Crystal structures obtained from PDB with sequence similarity $>50 \%$. ORF numbers in bold have been previously characterized in $P$. furiosus, those in italics were characterized by metal-targeted purification.

10 clusters were defined after hierarchical clustering and each cluster was manually evaluated. Six of the clusters were found to contain proteins exhibiting local agreement of MS/MS data to the Mo ICP-MS data and 12 such proteins were found in total (Table 3, Additional File 9). A novel Mo-protein (PF1587) was purified by the metalbased chromatography method from which the data set was derived [6] and this is identified by the GMPA analysis as a likely Mo-protein. This nicely demonstrates the effectiveness of the method given sufficient observation. Finally, comparing the results of the IPM analysis to the manually evaluated GMPA analysis derived results, among the 22 manually evaluated targets deemed to be likely Ni-proteins, only 3 had a Ni IPM match with 10 additional proteins having $\mathrm{Co}, \mathrm{Fe}, \mathrm{Mn}$ and $\mathrm{Zn}$ matches. Of the 20 manually evaluated targets deemed to be likely Mo-proteins, only 1 had a Mo IPM match.

Bioinformatic analyses of predicted $\mathrm{Ni}$ - and Mo-proteins As discussed above, 7 of the 22 genes listed in Table 2 encode proteins or subunits of proteins which have been shown to contain $\mathrm{Ni}$ ions in P. furiosus. This includes subunits of soluble hydrogenase I (SHI) and soluble hydrogenase 2 (SHII) grouped in clusters 7 and 8 respectively. In addition, PF0615 in cluster 13 is annotated as a hypA protein, which is implicated in Ni insertion in the hydrogenases. The structure of a hypA homolog from Thermococcus kodakaraensis has been solved and its Ni-binding site described [21]. This demonstrates that cluster 13 has at least two Ni-binding proteins that frequently co-occur in the fractionation space. Of the five ORFs in Table 2 with homologs whose crystal structures have been solved bound to metals other than $\mathrm{Ni}$, three are now known to bind $\mathrm{Ni}$ (PF0056 and PF0086) in P. furiosus or are known to have a Ni-binding site (PF0615). In particular PF0086 has been shown to bind $\mathrm{Ni}$ [6], but its homolog from the closely related Pyrococcus horikoshii (PDB 2E18) was expressed recombinantly and crystallized in a $\mathrm{Zn}$-bound form. This illustrates the flexibility of metal binding domains [22], and their ability to bind biologically incorrect metals when expressed heterologously [23]. The two remaining ORFs with non-Ni homolog crystal structures are PF1664, which contains the cysteines that bind 
Table 3 Manually Evaluated Molybdenum Protein Candidates

\begin{tabular}{|c|c|c|c|c|c|}
\hline Cluster & ORF & Annotation & $\begin{array}{c}\text { Crystal } \\
\text { structure } \\
\text { homolog }\end{array}$ & $\begin{array}{l}\text { Metal in } \\
\text { structure }\end{array}$ & $\begin{array}{l}\text { IPM } \\
\text { Metal }\end{array}$ \\
\hline 1 & PF0009 & ThiF family protein & 1JWB & $\mathrm{Zn}$ & \\
\hline 1 & PF0187 & Putative cofactor synthesis protein & & & $\mathrm{Fe}, \mathrm{Mo}, \mathrm{W}$ \\
\hline 1 & PF0668 & YjgF-like protein & 2DYY & & \\
\hline 1 & PF1718 & Wyosine base formation, Radical SAM & $2 Y X 0$ & & $\mathrm{Fe}$ \\
\hline 1 & PF1766 & Cell division transporter FtsY & 3DMD & & \\
\hline 2 & PF1956 & Fructose-1,6-bisphosphate aldolase class I & $10 J X$ & & \\
\hline 3 & PF1828 & Protein of unknown function DUF1621 & & & \\
\hline 3 & PF1886 & Carbohydrate/purine kinase & & & \\
\hline 4 & PF0098 & NAD+ synthase & $2 \mathrm{E} 18$ & & \\
\hline 4 & PF0236 & Phosphoribosyl pyrophosphokinase & $1 \cup 9 Y$ & & $\mathrm{Mg}$ \\
\hline 4 & PF1401 & Peptidyl-prolyl cis-trans isomerase & $11 \times 5$ & & \\
\hline 4 & PF1675 & Asp/Glu/hydantoin racemase & 2ZSK & & \\
\hline 4 & PF1731 & Signal recognition particle 54 & 3DM5 & & \\
\hline 5 & PF1538 & Amidohydrolase 1 & $1 \mathrm{P} 1 \mathrm{M}$ & $\mathrm{Ni}$ & \\
\hline 7 & PF0523 & Protein of unknown function DUF509 & 1ZD0 & $\mathrm{Mg}$ & \\
\hline 7 & PF1222 & Protein of unknown function DUF217 & & & \\
\hline 8 & PF1587 & Protein of unknown function DUF89 & $2 \mathrm{G} 8 \mathrm{~L}$ & & \\
\hline 9 & PF0212 & DNA polymerase, family B & $2 J G U$ & $\mathrm{Mn}$ & \\
\hline 9 & PF0306 & Translation factor, SUA5 type & & & \\
\hline 9 & PF0463 & Phosphoglycolate phosphatase & & & \\
\hline
\end{tabular}

Cluster number refers to hierarchical clustering with dynamic hybrid partitioning, see Figure 4 for explanation and supplementary tables 1-8 in Additional File 9 for complete cluster tables. Crystal structures obtained from PDB with sequence similarity $>50 \%$. ORF numbers in bold have been previously characterized in $P$. furiosus, those in italics were characterized by metal-targeted purification.

$\mathrm{Zn}$ (Cd in the crystal) in its homolog [24] and may be involved in binding Ni in P. furiosus, and PF2038 with a homolog that binds Mg-ATP. The only protein listed in Table 2 that is likely not to contain Ni is PF1861. This was previously purified from $P$. furiosus biomass and contained $\mathrm{Co}$ and $\mathrm{Zn}$ but not $\mathrm{Ni}$ [25]. This leaves 13 proteins that are predicted to contain $\mathrm{Ni}$. These proteins have no known or conjecturable $\mathrm{Ni}$ associations based on their sequences and are assumed to predominantly contain a set of undiscovered Ni-binding sites. Finally, it is worth pointing out that PF0056 is one of five ORFs (PF0144, PF1987, PF0056, PF0138 and PF1500) annotated as either conserved hypothetical or with only domain/motif matches and has now been shown to bind Ni [6].

In contrast to the case of $\mathrm{Ni}$, the pool of known molybdo-proteins in P. furiosus is small and far less can be ascertained bioinformatically. In particular, the role of Mo-proteins in P. furiosus is unclear, with only two such metalloproteins having recently been identified [6]. Consequently, only two of 20 proteins in Table 3 have either been shown to bind Mo (PF1587) or have an IPM hit for Mo (PF0187). The other recently identified Mo-protein that was purified from P. furiosus (PF1972) was observed in only 17 chromatography fractions and was rejected by the GMPA analysis, which depends on sufficient levels of occurrence in the data set to establish significance of metal-protein association. On the positive side, many of the uncharacterized proteins contain residues that could be involved in Mo-binding (e.g. 14 of 20 contain at least one cysteine residue as is often involved in Mo-pterin binding) [26-28], but given the extent and complexity of typical molybdopterin binding interactions and biochemistry [29] and the lack of knowledge of Mo-binding in organisms closely related to P. furiosus, we have not looked into this aspect further. On the negative side, DNA polymerase (Mo cluster 9 , Table 3 ), which has been well studied in many different organisms and is not known to bind or utilize Mo (although it is not clear if this has been directly considered previously) was picked as a top candidate Mo-protein. This illustrates that some of the targets that appear to reliably co-occur with a metal may be coincidental, or the result of interaction natively with additional proteins that are not strictly required for their primary function. Interestingly, four of the 20 predicted Mo-protein candidates have annotations that include "domain of unknown function." The confirmation of Mo-binding, which has 
already occurred for PF1587 by metal-directed purification, should provide an improved foundation for functionally characterizing these conserved domains which so far have been elusive [6].

\section{Known limitations}

We initially attempted to use standard correlation-based statistical techniques such as principal component analysis (PCA) and canonical correlation analysis (CCA) to determine associations between metals and proteins based on the experimental data that were available [6]. However, these efforts were hindered by the relatively non-quantitative nature of the MS/MS data available (lacking even spectral count information). Consequently, the GMPA analysis method was developed which is less reliant on quantitative agreement between these data sets. Simulated data sets demonstrated the effectiveness of GMPA scores alone given adequate separation regardless of the amount of noise observed in the peptide counts, but it was discovered that the metal-based fractionation typically did not produce a comprehensive enough data set containing an appropriate degree of overall separation. For example, the experimental data set is most consistent and comprehensive at the second column level (termed C2 in [7]) and separation is still relatively incomplete at this level. Consequently significance cut-off curves and clustering were employed completing the overall GMPA analysis methodology. It is likely that the predictive power of the methodology could be greatly improved by utilizing a data set with a more comprehensive fractionation across all levels, through the use of more quantitative MS/MS techniques [30-32] and more powerful statistical techniques (PCA/ CCA) that could then be applied more easily. This methodology could also potentially be carried out in a more automated fashion on an analytical scale to provide a rapid determination of the metalloproteins of any organism.

\section{Conclusions}

We have presented a computational methodology that can uncover probable metal-containing proteins using data from a non-comprehensive native fractionation coupled with metal and protein measurement using ICP-MS and MS/MS. This methodology has identified a number of candidate novel metalloproteins that are targets for future experimental verification. Application of the method to simulated data sets indicates that additional predictive accuracy could be achieved through the use of a more comprehensive fractionation. Our results for each of the 10 metals examined in this study underscore the unexplored complexity of metalloproteomes and have broad implications for protein structure and function as well as metal toxicity.

\section{Additional material}

\section{Additional file 1: Data infrastructure additional details. A more}

detailed description of the data infrastructure.

Additional file 2: Relational database schema figure. A diagram illustrating the basic layout of the relational database.

Additional file 3: OLAP cube figure. A diagram illustrating the basic connections between measure groups and dimensions in the OLAP cube.

Additional file 4: Relational database schema description.

Additional file 5: OLAP schema. The complete schema for the OLAP cube as an XML based backup.

Additional file 6: Significance curves, function calls for clustering Significance curves and parameters used to generate all clusterings, clusters.

Additional file 7: Cluster Diagrams. Tree diagrams of clusters. The colors are used simply to distinguish the defined clusters.

Additional file 8: Cluster Tables. Supplementary tables 1-8 containing complete cluster details.

Additional file 9: GMPA scores/occurrences tables. GMPA scores for all metals and proteins studied. The number of fraction occurrences in the data set is listed for each protein as well.

\section{Abbreviations}

The abbreviations used are: Co: cobalt; Fe: iron; Mn: manganese; Mo: molybdenum; Ni: nickel; Pb: lead; U: uranium; V: vanadium; W: tungsten; Zn: zinc; HT-MS/MS: high-throughput tandem mass spectrometry; ICP-MS: inductively coupled plasma mass spectrometry; ID: identifier; MS/MS: tandem mass spectrometry; OLAP: online analytical processing; ORF: open reading frame.

\section{Acknowledgements}

This work conducted by ENIGMA was supported by the Office of Science, Office of Biological and Environmental Research, of the U. S. Department of Energy under Contract No. DE-AC02-05CH11231.

\section{Author details}

${ }^{1}$ Department of Biochemistry and Molecular Biology, University of Georgia, Athens, GA 30602, USA. ²Philadelphia College of Osteopathic Medicine, Suwanee, GA 30024, USA. ${ }^{3}$ Scripps Center for Mass Spectrometry and the Departments of Molecular Biology and Chemistry, The Scripps Research Institute, La Jolla, CA, 92037, USA. ${ }^{4}$ Life Sciences Division, Lawrence Berkeley National Laboratory, Berkeley, CA 94720, USA.

\section{Authors' contributions}

WAL developed the bioinformatic metalloprotein prediction approach. JLP developed the OLAP infrastructure, data explorer software and Global Metal Protein Association analysis approach and manually evaluated the Mo and Ni clusters. WAL and JLP performed and interpreted the results of bioinformatics analyses of manually evaluated candidates. FLP, WAL and JLP developed the database infrastructure. JLP, FLP and WAL developed the ENIGMA IMPACT website. FLP and JLP wrote data loaders and loaded MS/ MS and chromatographic fractionation data into the database. FLP and JLP wrote ICP-MS data loading software and AC loaded all ICP-MS data and defined all metal peaks manually using this software. AC performed ICP-MS analyses. SAT, EK, JVA and GS performed HT-MS/MS analyses. AC, ALM, MPT and JWS grew and fractionated $P$. furiosus. AC, ALM, FEJ, FLP, JWS, MPT and JAT and MWWA developed the original metal-directed purification. JLP, MWWA and WAL wrote the manuscript. All authors read and approved the final manuscript.

Received: 10 October 2010 Accepted: 28 February 2011 Published: 28 February 2011

\section{References}

1. Mounicou S, Szpunar J, Lobinski R: Metallomics: the concept and methodology. Chemical Society Reviews 2009, 38:1119-1138. 
2. Waldron KJ, Rutherford JC, Ford D, Robinson NJ: Metalloproteins and metal sensing. Nature 2009, 460:823-830.

3. Andreini C, Bertini I, Rosato A: Metalloproteomes: A Bioinformatic Approach. Acc Chem Res 2009, 42:1471-1479.

4. Dupont CL, Butcher A, Valas RE, Bourne PE, Caetano-Anolles G: History of biological metal utilization inferred through phylogenomic analysis of protein structures. Proc Natl Acad Sci USA 2010, 107:10567-10572.

5. Waldron KJ, Robinson NJ: How do bacterial cells ensure that metalloproteins get the correct metal? Nat Rev Microbiol 2009, 7:25-35.

6. Cvetkovic A, Menon AL, Thorgersen MP, Scott JW, Poole FL, Jenney FE Jr, Lancaster WA, Praissman JL, Shanmukh S, Vaccaro BJ, et al: Microbial metalloproteomes are largely uncharacterized. Nature 2010, 466:779-782

7. Menon AL, Poole FL, Cvetkovic A, Trauger SA, Kalisiak E, Scott JW, Shanmukh S, Praissman J, Jenney FE Jr, Wikoff WR, et al: Novel multiprotein complexes identified in the hyperthermophilic archaeon Pyrococcus furiosus by non-denaturing fractionation of the native proteome. Mol Cell Proteomics 2009, 8:735-751.

8. Haferburg G, Kothe E: Metallomics: lessons for metalliferous soil remediation. Appl Microbiol Biotechnol 2010, 87:1271-1280.

9. Tottey S, Waldron KJ, Firbank SJ, Reale B, Bessant C, Sato K, Cheek TR, Gray J, Banfield MJ, Dennison C, Robinson NJ: Protein-folding location can regulate manganese-binding versus copper- or zinc-binding. Nature 2008, 455:1138-1142

10. Sevcenco AM, Krijger GC, Pinkse MW, Verhaert PD, Hagen WR, Hagedoorn PL: Development of a generic approach to native metalloproteomics: application to the quantitative identification of soluble copper proteins in Escherichia coli. J Biol Inorg Chem 2009, 14:631-640.

11. Kehl C, Simms AM, Toofanny RD, Daggett V: Dynameomics: a multidimensional analysis-optimized database for dynamic protein data. Protein Eng Des Sel 2008, 21:379-386.

12. Hunter S, Apweiler R, Attwood TK, Bairoch A, Bateman A, Binns D, Bork P, Das $U$, Daugherty $L$, Duquenne $L$, et al: InterPro: the integrative protein signature database. Nucleic Acids Res 2009, 37:D211-215.

13. $R$ : A language and environment for statistical computing. $R$ Foundation for Statistical Computing, Vienna, Austria. ISBN 3-900051-07-0. [http:// www.R-project.org].

14. Langfelder $\mathrm{P}$, Zhang $\mathrm{B}$, Horvath $\mathrm{S}$ : Defining clusters from a hierarchical cluster tree: the Dynamic Tree Cut package for R. Bioinformatics 2008, 24:719-720.

15. Zhang B, Horvath S: A general framework for weighted gene coexpression network analysis. Stat Appl Genet Mol Biol 2005, 4:Article17.

16. Chvatal V: A Greedy Heuristic for the Set-Covering Problem. Mathematics of operations research 1979, 4:233-235.

17. Andreini C, Bertini I, Cavallaro G, Holliday GL, Thornton JM: Metal ions in biological catalysis: from enzyme databases to general principles. Journal Of Biological Inorganic Chemistry 2008, 13:1205-1218.

18. Yamaguchi A, lida K, Matsui N, Tomoda S, Yura K, Go M: Het-PDB Navi.: a database for protein-small molecule interactions. J Biochem 2004, 135:79-84.

19. Xiao Z, Wedd AG: The challenges of determining metal-protein affinities. Nat Prod Rep 2010, 27:768-789.

20. Pruitt KD, Tatusova T, Maglott DR: NCBI reference sequences (RefSeq): a curated non-redundant sequence database of genomes, transcripts and proteins. Nucleic Acids Res 2007, 35:D61-65.

21. Watanabe $S$, Arai T, Matsumi R, Atomi H, Imanaka T, Miki K: Crystal structure of HypA, a nickel-binding metallochaperone for [NiFe] hydrogenase maturation. J Mol Biol 2009, 394:448-459.

22. Dudev T, Lim C: Metal Binding Affinity and Selectivity in Metalloproteins: Insights from Computational Studies. Annual Review of Biophysics 2008, 37:97-116

23. Jenney FE Jr, Adams MW: Rubredoxin from Pyrococcus furiosus. Methods Enzymol 2001, 334:45-55.

24. Sivaraman J, Myers RS, Boju L, Sulea T, Cygler M, Jo Davisson V, Schrag JD: Crystal structure of Methanobacterium thermoautotrophicum phosphoribosyl-AMP cyclohydrolase Hisl. Biochemistry 2005, 44:10071-10080

25. Story SV, Shah C, Jenney FE Jr, Adams MW: Characterization of a novel zinc-containing, lysine-specific aminopeptidase from the hyperthermophilic archaeon Pyrococcus furiosus. J Bacteriol 2005, 187:2077-2083.
26. Brondino CD, Romao MJ, Moura I, Moura JJ: Molybdenum and tungsten enzymes: the xanthine oxidase family. Curr Opin Chem Biol 2006, 10:109-114.

27. Gladyshev VN, Khangulov SV, Axley MJ, Stadtman TC: Coordination of selenium to molybdenum in formate dehydrogenase $\mathrm{H}$ from Escherichia coli. Proc Natl Acad Sci USA 1994, 91:7708-7711.

28. Romao MJ, Knablein J, Huber R, Moura JJ: Structure and function of molybdopterin containing enzymes. Prog Biophys Mol Biol 1997, 68:121-144.

29. Schwarz G, Mendel RR, Ribbe MW: Molybdenum cofactors, enzymes and pathways. Nature 2009, 460:839-847.

30. Lu P, Vogel C, Wang R, Yao X, Marcotte EM: Absolute protein expression profiling estimates the relative contributions of transcriptional and translational regulation. Nat Biotechnol 2007, 25:117-124.

31. Silva JC, Gorenstein MV, Li GZ, Vissers JP, Geromanos SJ: Absolute quantification of proteins by LCMSE: a virtue of parallel MS acquisition Mol Cell Proteomics 2006, 5:144-156.

32. Zhu W, Smith JW, Huang CM: Mass spectrometry-based label-free quantitative proteomics. J Biomed Biotechnol 2010, 2010:840518.

doi:10.1186/1471-2105-12-64

Cite this article as: Lancaster et al: A Computational Framework for Proteome-Wide Pursuit and Prediction of Metalloproteins using ICP-MS and MS/MS Data. BMC Bioinformatics 2011 12:64.

\section{Submit your next manuscript to BioMed Central and take full advantage of:}

- Convenient online submission

- Thorough peer review

- No space constraints or color figure charges

- Immediate publication on acceptance

- Inclusion in PubMed, CAS, Scopus and Google Scholar

- Research which is freely available for redistribution

Submit your manuscript at www.biomedcentral.com/submit
Ciomed Central 